Tissue Engineering and Delivery Systems

Biotechnology and Bioengineering DOI 10.1002/bit.25589

\title{
Fibrin Gels Engineered with Pro-Angiogenic Growth Factors Promote Engraftment of Pancreatic Islets in Extrahepatic Sites in Mice ${ }^{\dagger}$
}

Mejdi Najjar ${ }^{1}$, Vita Manzoli ${ }^{1,7}$, Chiara Villa ${ }^{1,8,9}$, Mikaël M. Martino ${ }^{2}$, R. Damaris Molano ${ }^{1}$, Yvan Torrente $^{1,8,9}$, Antonello Pileggi ${ }^{1,3,4,5}$, Luca Inverardi ${ }^{1,4,6}$, Camillo Ricordi ${ }^{1,3,4,5,6}$, Jeffrey A. Hubbell ${ }^{1,2}$, Alice A. Tomei ${ }^{1,7,3^{*}}$

${ }^{1}$ Diabetes Research Institute, University of Miami Miller School of Medicine, Miami, FL

${ }^{2}$ Institute of Bioengineering, Ecole Polytechnique Fédérale de Lausanne (EPFL), Lausanne,

Switzerland

${ }^{3}$ Department of Surgery, University of Miami Miller School of Medicine, Miami, Florida, USA

${ }^{4}$ Department of Microbiology and Immunology, University of Miami Miller School of Medicine, Miami, Florida, USA

${ }^{5}$ Department of Biomedical Engineering, University of Miami, Miami, Florida, USA

${ }^{6}$ Department of Medicine, University of Miami Miller School of Medicine, Miami, Florida, USA

${ }^{7}$ Department of Electronics, Information and Bioengineering, Politecnico di Milano, Italy

${ }^{8}$ Department of Pathophysiology and Transplantation, Universitá degli Studi di Milano, Italy

${ }^{9}$ Fondazione IRCCS Ca' Granda Ospedale Maggiore Policlinico, Italy

*Corresponding author: Alice A. Tomei, 1450 NW 10 ${ }^{\text {th }}$ Avenue, Miami, FL-33136; Phone: +1 305-

243-3469; Email: atomei@med.miami.edu

Running Title: Engineered Gels for Islet Transplantation

${ }^{\dagger}$ This article has been accepted for publication and undergone full peer review but has not been through the copyediting, typesetting, pagination and proofreading process, which may lead to differences between this version and the Version of Record. Please cite this article as doi: [10.1002/bit.25589]

Additional Supporting Information may be found in the online version of this article.

This article is protected by copyright. All rights reserved

Received October 24, 2014; Revision Received January 21, 2015; Accepted March 9, 2015

This article is protected by copyright. All rights reserved 


\section{Abstract}

With a view toward reduction of graft loss, we explored pancreatic islet transplantation within fibrin matrices rendered pro-angiogenic by incorporation of minimal doses of vascular endothelial growth factor-A165 and platelet-derived growth factor-BB presented complexed to a fibrin-bound integrinbinding fibronectin domain. Engineered matrices allowed for extended release of pro-angiogenic factors and for their synergistic signaling with extracellular matrix-binding domains in the posttransplant period. Aprotinin addition delayed matrix degradation and prolonged pro-angiogenic factor availability within the graft. Both subcutaneous (SC) and epididymal fat pad (EFP) sites were evaluated. We show that in the SC site, diabetes reversal in mice transplanted with 1,000 IEQ of syngeneic islets was not observed for islets transplanted alone, while engineered matrices resulted in a diabetes median reversal time (MDRT) of 38 days. In the EFP site, the MDRT with 250 IEQ of syngeneic islets within the engineered matrices was 24 days, versus 86 days for islets transplanted alone. Improved function of engineered grafts was associated with enhanced and earlier (by day 7) angiogenesis. Our findings show that by engineering the transplant site to promote prompt revascularization, engraftment and long-term function of islet grafts can be improved in relevant extrahepatic sites. This article is protected by copyright. All rights reserved

Keywords: islet transplantation, angiogenesis, hydrogels, local release, PDGF-BB, VEGF-A 


\section{Introduction}

Transplantation of pancreatic islets is less invasive than full pancreas transplantation and may cure type 1 diabetes mellitus (T1DM) (Harlan et al. 2009; Mineo et al. 2009). Currently, islets isolated from the pancreas of cadaveric donors are clinically transplanted intrahepatically (Mineo

et al. 2009). Insulin independence at one-year post transplant is achieved in approximately $60 \%$ of the patients and in most cases is diminished over time due to graft loss (Mineo et al. 2009). Early graft loss amounts to $50-75 \%$ of the transplanted islet mass and is mainly due to the suboptimal environment provided by the liver transplant site (Cantarelli and Piemonti 2011). Among the influences that can cause early graft loss is the poor re-vascularization that in turn causes lack of glucose homeostasis and hypoxia, in addition to the higher concentration of toxic immunosuppressive drugs in the portal vein that can impair angiogenesis and islet proliferation (Cantarelli and Piemonti 2011).

Alternative transplant sites have been long sought to improve islet engraftment and long-term function (Cantarelli and Piemonti 2011). The choice of alternative sites aims at minimizing early inflammatory reactions, promoting a well vascularized microenvironment (to guarantee exchange of oxygen and nutrients, $\mathrm{pH}$ homeostasis and cell waste removal) and gaining easyaccess to the transplant site (for minimally invasive surgery and follow-up) (Cantarelli and Piemonti 2011). Among the sites that have been explored are the subcutaneous (SC) site (Barkai et al. 2012; Juang et al. 1996; Kim et al. 2012; Nishimura et al. 2013; Pileggi et al. 2006) and the omentum (Ao et al. 1993; Berman et al. 2009; Gustavson et al. 2005; Jacobs-TulleneersThevissen et al. 2010; Kin et al. 2003; Kriz et al. 2012; Pedraza et al. 2012).

This article is protected by copyright. All rights reserved 
We focused our attention on optimization of islet engraftment in the SC tissue and the epididymal fat pad (EFP), as a surrogate of the omental pouch, in the murine model. The SC site is easily accessible and offers an extensive surface area for transplantation. However, results in the SC site have been disappointing, for reasons that may include poor oxygen tension, slow revascularization, inflammation and need for mechanical protection (Beattie et al. 2002; Gu et al. 2001; Juang et al. 2005; Pileggi et al. 2006; Wang et al. 2002). The omental pouch site is well vascularized and provides portal drainage, which is desirable for physiological effects of insulin on the liver, and it can accommodate large graft volumes (Ao et al. 1993; Berman et al. 2009; Gibly et al. 2011; Gustavson et al. 2005; Jacobs-Tulleneers-Thevissen et al. 2010; Kim et al. 2010; Kin et al. 2003; Kriz et al. 2012; McQuilling et al. 2011; Pedraza et al. 2012).

Devices and scaffolds for engineering the islet transplant microenvironment provide mechanical support and allow promoting angiogenesis and improving islet engraftment and long-term function in extrahepatic sites (Barkai et al. 2012; Colton 1995; Juang et al. 1996; Kim et al. 2012; Marzorati et al. 2009; Pedraza et al. 2012; Pileggi et al. 2006). However, non-degradable biomaterials can generate foreign body responses that impair islet function and can trigger graft rejection. Alternatively, fibrin matrices are natural scaffolds that provide transient mechanical support and completely degrade days after implantation through physiological cell-mediated proteolytic mechanisms leading to ingrowth of autologous tissues, including vascular beds, as the result of host remodeling. In addition, fibrin matrices have been shown to be beneficial for islet culture (Beattie et al. 2002).

This article is protected by copyright. All rights reserved 
In a wound healing model we have previously shown that covalently incorporating a multifunctional recombinant fibronectin (FN) fragment that contains (i) a transglutaminase substrate for covalent binding to fibrin under the enzymatic action of Factor XIIIa, (ii) the major integrin-binding domain (FNIII 9-10), and (iii) the growth factor (GF)-binding domain FNIII 1214, (together referred to as FNIII 9-10/12-14) within fibrin gels can present incorporated proangiogenic GFs (vascular endothelial GF, VEGF-A165, and platelet-derived GF, PDGF-BB) in a manner to yield potent and functional angiogenesis with minimal doses of GFs (improving safety and cost-effectiveness) (Eming and Hubbell 2011; Martino and Hubbell 2010; Martino et al. 2009; Martino et al. 2011). Potentiation of GF regenerative effects result from co-association of the integrin $\alpha_{5} \beta_{1}$ with VEGF-receptor 2 or PDGF-receptor $\beta$ by proximal binding of the respective GF to the FNIII12-14 domain, which resides approximately $5 \mathrm{~nm}$ from the FNIII9-10 integrin-binding domain in the FNIII 9-10/12-14 recombinant protein, leading to a potent synergist signaling and morphogenesis with doses of GFs that are ineffective if delivered alone (Martino et al. 2011). Here we show that engineering the SC and EFP sites with FNIII9-10/1214-functionalized fibrin matrices containing aprotinin (which delays matrix degradation and prolongs pro-angiogenic GF availability within the graft) presenting VEGF-A165 and PDGF-BB accelerates reversal of diabetes with fewer islets than matrices without GFs and matrix-free conditions, and that this correlates with induction of prompt re-vascularization in syngeneic murine islet transplant models.

\section{Materials and Methods}

\section{Engineered Fibrin Gels}

Fibrin matrices were formed as described previously (Martino et al. 2009). Briefly, human 
fibrinogen depleted of fibronectin, plasminogen, and von Willebrand factor (Enzyme Research Laboratories, South Bend, IN, USA) at $8 \mathrm{mg} / \mathrm{ml}$ and supplemented with or not (w/o APR) 17 $\mu \mathrm{g} / \mathrm{ml}$ aprotinin (Sigma-Aldrich) was cross-linked with $2 \mathrm{U} / \mathrm{ml}$ human thrombin (Sigma-Aldrich) and $8 \mathrm{U} / \mathrm{ml}$ factor XIIIa (Fibrogammin; Behring, Marburg, Germany) in $2.5 \mathrm{mM}$ calcium chloride in HEPES buffer. FN III9-10/12-14 was produced as described to comprise a factor XIIIa substrate at the $\mathrm{N}$ terminus, consisting of residues $1-8$ of the protein $\mathrm{alpha}_{2}$ plasmin inhibitor ( $\mathrm{a}_{2} \mathrm{PI}_{1-8}, \mathrm{NQEQVSPL}$ ): $\mathrm{a}_{2} \mathrm{PI}_{1-8}-\mathrm{FN}$ IIII9-10/12-14 (Martino et al. 2011). $\mathrm{a}_{2} \mathrm{PI}_{1-8}-\mathrm{FN}$ III910/12-14 was covalently incorporated in $80 \mu \mathrm{l}$ (SC grafts) or $20 \mu \mathrm{l}$ (EFP grafts) fibrin gels at 2 $\mu \mathrm{M}$ (ISL+FIB). For experimental conditions containing GFs ('ISL+FIB+GFs'), hPDGF-BB (2500 ng/ml; $50 \mathrm{ng}$ in the EFP site or $200 \mathrm{ng}$ in the SC site, carrier-free from R\&D Systems) and hVEGF-A165 (5000 ng/ml; $100 \mathrm{ng}$ in the EFP site or $400 \mathrm{ng}$ in the SC site, carrier-free from R\&D Systems, Minneapolis, MN) were also incorporated.

\section{Islet Transplantation in the Subcutaneous Site}

At the time of islet transplantation, aliquots of $1000 \mathrm{IEQ} /$ each were collected with a precision syringe (Hamilton; Reno, NV) and transferred to a low-binding $0.5 \mathrm{ml}$ microfuge tube (Eppendorf; Hauppauge, NY). After centrifugation at 1200 rpm, 2 min, RT, residual supernatant was removed and islets were resuspended within $80 \mu$ l of engineered fibrin gels (without GFs for 'ISL+FIB mix'; with hVEGF-A165 and hPDGF-BB for 'ISL+FIB+GF mix' and 'ISL+FIB+GF w/o APR mix' experimental groups), and then immediately transferred to a sterile metal spatula for gelation to occur (Figure 1A). The resulting islets-containing fibrin gels measured approximately $5 \times 10 \times 1 \mathrm{~mm}$. For analysis of angiogenesis at day 7 and day 21 after transplant, 450 IEQ islets were resuspended in $32 \mu 1$ gels.

This article is protected by copyright. All rights reserved 
Under general anesthesia (isoflurane USP; Baxter, Deerfield, IL), a small cutaneous incision was performed on the abdomen of recipient mice. The skin was gently separated from the muscle underneath to create a subcutaneous pocket to accommodate the islet-containing fibrin gels. Control islets alone, without a fibrin matrix ('ISL AL'), were collected with the precision syringe and directly injected in the SC pocket. The skin was sutured with absorbable sutures (Ethicon; Somerville, NJ).

Experimental Groups are summarized in Table I.

\section{Islet Transplantation in the Epididymal Fat Pad Site}

Under general anesthesia, small cutaneous and muscular incisions were performed on the abdomen of recipient mice. The epididymal fat pad (EFP) was then gently exposed and flattened on a sterile gauze surface. At the time of islet transplantation, from aliquots of 250 (for optimization of syngeneic transplants) or 1000 (for characterization of syngeneic transplants at day 7, 14 and 21 after transplant) or 750 / 1000 / 1200 (for allogeneic transplants) IEQ/each, islets were collected with a precision syringe. For the 'ISL AL' experimental group, islets were transferred to the surface of the EFP and well distributed on top of the pad. For 'ISL+FIB top' (without GFs) and 'ISL+FIB+GF top' (with hVEGF-A165 and hPDGF-BB) experimental groups, islets collected with the syringe were transferred to the surface of the EFP and well distributed on top of the pad. Then $20 \mu \mathrm{l}$ of engineered fibrin gels were pipetted on the EFP to cover the islets and secure their direct contact with EFP host blood vessels. Gelation was allowed to occur in situ. Finally, for 'ISL+FIB mix' (without GFs) and 'ISL+FIB+GF mix' (with hVEGF-A165 and hPDGF-BB) experimental groups, islets collected with the syringe were transferred to a low-binding $0.5 \mathrm{ml}$ microfuge tube. Islet residual supernatant was removed and 
islets were resuspended within $20 \mu \mathrm{l}$ of engineered fibrin gels and immediately transferred to the EFP for gelation to occur in situ. The EFP was then wrapped and fibrin gel was used to seal the 'EFP pocket'. Once the gel had polymerized the wrapped islet-containing EFP was gently placed back in the abdominal cavity of the mouse and the muscle and the skin were sutured with absorbable sutures.

Experimental Groups are summarized in Table I.

This article is protected by copyright. All rights reserved 


\section{Results}

\section{Engineered fibrin matrix containing growth factors allows engraftment and long-term}

\section{function of islets transplanted in the subcutaneous site}

For SC transplantation, fibrin gels measured approximately $5 \times 10 \times 1 \mathrm{~mm}^{3}$ with a volume of $80 \mu 1$ (Figure 1A). A dose of 1000 IEQ syngeneic islets transplanted alone in the SC site reverted diabetes only in $20 \%$ of mice after 100 days (undefined median reversal time (MRT), n=5)

(Figure 1B-C, black). Islets transplanted in the SC site within fibrin matrices engineered for potentiated delivery of the proangiogenic factors VEGF-A and PDGF-BB (ISL+FIB+GF mix) and containing aprotinin reverted diabetes in $60 \%$ of mice at 40 days post-transplant (MRT: 38 days, $n=5$ ) (Figure 1B, red). In these mice blood glucose was controlled more tightly (Figure 1D red) than with islets transplanted with islets alone (without a fibrin matrix or GFs) (ISL AL, Figure 1C). In the absence of GFs, no beneficial role was observed for transplantation of islets within fibrin matrices containing the bound FNIII 9-10/12-14 protein (ILS+FIB, undefined MRT, n=5) (Figure 1B-E, green). In the absence of aprotinin (ISL+FIB+GF w/o APR mix), a delay in diabetes reversal time was observed (MRT: 84 days, n=3) (Figure 1B-F, pink) suggesting that matrix stability in vivo (resulting from the presence of aprotinin) is critical for exploiting the beneficial effect of matrix-bound GFs co-presented with integrin-binding sites (ISL+FIB+GF mix) (Figure 1E). We conclude that aprotinin-containing engineered fibrin matrix supplemented with GFs (ISL+FIB+GF mix) improves engraftment and as consequence it accelerates function of islets transplanted in the SC site through an effect of GF delivery via the engineered matrix.

This article is protected by copyright. All rights reserved 
Engineered fibrin matrix containing growth factors promotes early re-vascularization of islet transplants in the subcutaneous site

Syngeneic pancreatic islets were transplanted SC at $450 \mathrm{IEQ} /$ mouse to study the degree of engraftment by histopathology. Most islet alone and islets transplanted in the SC site within fibrin matrices but without GFs (ISL+FIB) did not survive after transplantation. Those few islets surviving showed poor re-vascularization at day 7 (Figure 2A-B), as indicated by the low expression of CD31 ${ }^{+}$Lyve- $1^{-}$blood and CD $31^{+}$Lyve $-1^{+}$lymphatic vessels (pie chart, black) within insulin ${ }^{+}$and glucagon ${ }^{+}$islets (pie chart, dark grey). On the other hand, islets transplanted in the SC site within fibrin matrices engineered for controlled presentation of VEGF-A and PDGF-BB (ISL+FIB+GF mix) and containing aprotinin survived transplantation in larger amounts and showed rich networks of CD $31^{+}$Lyve- $1^{-}$blood and CD $31^{+}$Lyve- $-1^{+}$lymphatic vessels at day 7 (Figure 2C) and day 21 (Figure 2D) within the islet grafts. Shown are representative images of surviving grafts. We conclude that engineered fibrin matrix promotes early ( $<7$ days) re-vascularization of islets after transplant in the SC site, correlating with the observed improvement in engraftment and long-term function of the transplanted islets. This is also in accordance with our previous findings in the wound healing model, where we found that the regenerative effects of pro-angiogenic GFs were enhanced when the GFs were delivered through fibrin gels engineered with the multifunctional recombinant fibronectin fragment (Martino et al. 2011).

Engineered fibrin matrix containing growth factors promotes endothelial and fibroblast-like cell outgrowth and proliferation from ex vivo cultured islets

This article is protected by copyright. All rights reserved 
Analysis at day 2, 3, 5, and 11 of phase contrast images of islets cultured ex vivo either alone (ISL AL), within fibrin gels (ISL+FIB), within fibrin gels with GFs with or without aprotinin (ISL+FIB+GF mix; ISL+FIB+GF w/o APR mix) showed that there was no difference in cell outgrowth (as percentage of total area occupied by fibroblast and endothelial-like cells growing on the culture dish and out of the islets) between islets cultured ex vivo either alone (ISL AL) or within fibrin gels (ISL+FIB, p>0.05) at day 2, 3, 5, and 11 after seeding (Figure 3B). At day 3, 5 and 11, we saw higher cell outgrowth for islets cultured ex vivo within fibrin gels containing VEGF-A and PDGF-BB with aprotinin (ISL+FIB+GF, p<0.01) and without aprotinin (ISL+FIB+GF w/o APR, p<0.05) compared to both ISL AL and ISL+FIB (Figure 3B). Finally, at day 5, higher cell outgrowth was observed for ISL+FIB+GF compared to ISL+FIB+GF w/o APR $(\mathrm{p}<0.05)$ (Figure 3B). Thus, the engineered fibrin matrix containing GFs promotes outgrowth of cells that are involved in promoting revascularization from islets, and inclusion of aprotinin in the matrix potentiates this, although the beneficial effects of aprotinin inclusion are more apparent in vivo.

\section{Engineered fibrin matrix containing growth factors promotes engraftment, re-vascularization} and function of islets transplanted in the epididymal fat pad site

A dose of 250 IEQ of islets transplanted alone in the EFP pouch reverted diabetes only in $10 \%$ of mice at 40 days after transplant (MRT: 86 days, $n=8$ ) (Figure 4A-B, black, $n=8$ ). When islets were transplanted within fibrin gels assembled ex vivo (ISL+FIB mix), rather than placed on top of EFP and covered by fibrin gels (ISL+FIB top), we observed no improvement in either diabetes reversal time or percentage of mice that reverted (MRT: undefined, $n=5, p<0.05$ ) (Figure 4A, green), or in blood glucose control (Figure 4C, green) even when GFs were added to the gel 
$($ ISL+FIB+GF mix) (MRT: undefined, $\mathrm{n}=5, \mathrm{p}>0.05)$ (Figure 4A-D, red). Islets transplanted in the EFP site and covered by fibrin matrices engineered for controlled presentation of VEGF-A and PDGF-BB (ISL+FIB+GF top) reverted diabetes in $60 \%$ of mice at 40 days post-transplant (MRT: 38 days, $\mathrm{n}=10$ (Figure 4A, blue) showing an improvement over ISL $A L(p \leq 0.05)$. In mice transplanted with ISL+FIB+GF top, blood glucose was controlled more tightly than in ISL AL (Figure 4E, blue). In the absence of GFs, we did not observe a beneficial role for islets transplanted in the EFP and covered with fibrin matrices containing the FNIII9-10/12-14 protein (ILS+FIB top, MRT: 54 days, $n=4$ ) compared to ISL AL (black, p>0.05) and ISL+FIB+GF top $(\mathrm{p}>0.05)$ as both percentage of mice that reversed diabetes (Figure 4A, orange) and control of blood glucose (Figure 4F, orange).

In all fibrin conditions, aprotinin was included, in consideration of its beneficial effects that we observed in the SC site. We conclude that the engineered fibrin matrix containing the GFs improves engraftment and long-term function of islets transplanted in the EFP site when islets are seeded in direct contact with the EFP and the matrix is polymerized on top.

Histological analysis of grafts retrieved at day 110 after transplant showed that islets transplanted in the EFP site and covered with fibrin matrices engineered for controlled presentation of VEGF-A and PDGF-BB (ISL+FIB+GF top) survived in large amounts (as INS ${ }^{+}$ area) and showed rich networks of CD $31^{+}$Lyve $-1^{-}$blood and CD $31^{+}$Lyve- $1^{+}$lymphatic vessels within and in proximity to insulin ${ }^{+}$and glucagon ${ }^{+}$islets (Figure 4G). Vascularization of islets transplanted in the ISL+FIB+GF top condition was comparable to islets within the native pancreas (Figure 4H, p>0.05).

Transplantation of 1000 IEQ/mouse islets with engineered fibrin gels in the optimized EFP site (ISL+FIB+GF top, blue) reverted diabetes in less than 24 hours after transplant (Figure 
5A). Histological analysis of grafts retrieved at day 7 (Figure 5B), 14 (Figure 5C), and 21

(Figure 5D) after transplant showed that ISL+FIB+GF top survived transplantation, maintained their phenotype (as distribution of insulin ${ }^{+}$beta cells and glucagon ${ }^{+}$alpha cells) and showed rich networks of $\mathrm{CD} 1^{+}$blood and lymphatic vessels within and in proximity to insulin ${ }^{+}$and glucagon $^{+}$islets as early as day 7 after transplant (Figure 5B-D). We conclude that the engineered fibrin matrix allows early ( $<7$ days) re-vascularization and maintenance of islet phenotype, correlating with the observed engraftment and function when islets are placed directly on the EFP and covered with the matrix.

Dosing studies in allograft models (C57BL/6 islets to fully MHC-mismatched diabetic BALB/c mice) showed that euglycemia could be reached by transplanting as few as 750 IEQ/mouse (Figure 5E, orange), but that higher percentages of diabetes reversal and better control of blood glucose could be achieved by transplanting 1000 (Figure 5E, red) or 1200 (Figure 5E, black) IEQ/mouse $(\mathrm{p}<0.001)$. The difference in islet requirements between the syngeneic and allogeneic models are consistent with previous work and mirror the more challenging host response when allogeneic immune attacks against the donor islets are present, leading to loss of the islet graft function and return to hyperglycemia between 9 and 14 days after transplant (Figure 5E).

\section{Discussion}

Islet transplantation restores metabolic control in people with diabetes with less morbidity and invasiveness than whole pancreas transplantation (Gill and Bishop 2012; Johnson and Jones 2012; Mineo et al. 2009; Rickels 2012; Shapiro 2011; Tavakoli and Liong 2012).

Although clinical protocols have been greatly improved during the past years, several issues that

This article is protected by copyright. All rights reserved 
concern islet sourcing, islet engraftment and long-term function still need to be addressed (Barshes et al. 2005; Cantarelli and Piemonti 2011; Mineo et al. 2009; Plesner and Verchere 2011; Rajab 2010; van der Windt et al. 2008). Intrahepatic islet transplantation is associated with early loss of functional islet mass, hypoxia and inflammation leading to poor engraftment (Barshes et al. 2005; Cantarelli and Piemonti 2011; Rajab 2010; van der Windt et al. 2008). Prompt neovascularization of transplanted islets is paramount for adequate engraftment.

We have sought to address these limitations by using engineered biomaterial matrices that improve islet engraftment through promotion of early re-vascularization in two extrahepatic sites: the SC (easy to access for implantation) and EFP (as a surrogate for the omental pouch in humans, since in mice the anatomy of the omentum is very different than in humans while the EFP recapitulates most features, like the cell composition and the amount of vascular beds) sites. We show here that we can reverse diabetes and maintain euglycemia earlier and in a higher proportion of recipients by transplanting islets with engineered fibrin matrices; we utilized fibrinbased matrices engineered for delivery of minimal doses of pro-angiogenic GFs in close proximity to integrin-binding domains and confirmed that such an approach can potentiate the regenerative potential of the GFs and enhance angiogenesis, consistent with our previous observations in a wound healing model (Martino et al. 2011). We chose to implant suboptimal numbers of islets in order to distinguish functional differences in islet performances (engraftment and long-term function) between the different experimental groups.

The need has been recognized for higher islet doses or hand-picking of smaller islets for mechanical protection to attain diabetes reversion in the SC site compared to other sites, including the renal subcapsular space and EFP site (Juang et al. 1996; Kim et al. 2012; MacGregor et al. 2006; Marzorati et al. 2009; Pileggi et al. 2006). Therefore, the rather high 
requirements of islet dose observed in our pilot work (Marzorati et al. 2009) and utilized here for achieving euglycemia even in the optimized model is in accordance with previous findings. Our results suggest that transplantation of islets within engineered fibrin matrices allows minimizing the islet dose to achieve normoglycemia even in such a challenging transplant site like the SC site. In the less challenging EFP site, by utilizing engineered fibrin matrices, we could obtain engraftment and long-term function with minimal doses of islets, especially when compared to other approaches for GF delivery trhough biomaterials. Also, in the EFP site, we observed better engraftment and long-term function of islets placed directly in contact with the well-vascularized EFP site and covered by fibrin scaffolds containing GFs (ISL+FIB+GF top) rather than with islets transplanted within fibrin scaffolds prepared ex vivo (ISL+FIB+GF mix), which we found to be beneficial in transplantation in the SC site. We hypothesize that this is because the graft in the SC site is subjected to higher mechanical stresses and hypoxic conditions than in the EFP site (Juang et al. 1996; Kim et al. 2012; MacGregor et al. 2006; Marzorati et al. 2009; Pileggi et al. 2006). In the SC site, diffusional limitations, which may arise from embedding the islets within a gel construct, may be outweighed by the higher need for mechanical support, and the beneficial effect of GF delivery through the engineered fibrin matrices that counterbalances the low density of host vessels. A beneficial effect of SC transplantation of islets embedded within hydrogels, including fibrin gels, is in agreement with previous findings (Kim et al. 2012; Phelps et al. 2013). On the other hand, in the EFP site, which is highly vascularized, islets received more benefit from being placed in direct contact with layers of highly vascularized tissue rather than dispersed within fibrin matrices. Addition of fibrin gels, and in particular gels engineered with GFs, on top of islets in the EFP site helps preserve direct interactions between islets and host vessels and prevents islet clumping, consistent with an overall beneficial effect of supporting matrices 
reported in previous studies (Juang et al. 1996; Kim et al. 2012; MacGregor et al. 2006;

Marzorati et al. 2009; Pileggi et al. 2006). Another possible interpretation is that the engineered fibrin matrix is too dense and does not degrade quickly enough for host vessel invasion to occur at a rate fast enough to support the survival of the marginal mass of islets that we transplanted in the EFP site, as suggested by previous work confirming that fibrin concentrations directly influences the rates of neovascularization. Although not formally addressed in our study, it is conceivable that the 'topping' implantation technique may contribute to improve islet engraftment also in the SC site. This approach may allow achieving direct contact of islets with at least one of the host's tissue surfaces from which oxygen, nutrients and new vascularization is received in the peritransplant period, therefore helping to reduce diffusion barriers in the ex vivo assembled scaffolds.

The deleterious role of hypoxia after islet transplantation and the beneficial effect of counteracting it have been reported (Stokes et al. 2013). Among proposed strategies are novel means to induce re-vascularization,(Brissova et al. 2006; Golocheikine et al. 2010; Hussey et al. 2009; Jalili et al. 2011; Lai et al. 2005; Ohmura et al. 2010; Rivas-Carrillo et al. 2006; Salvay et al. 2008; Uonaga et al. 2010; Zhang et al. 2004), several of which utilize VEGF (Brissova et al. 2006; Lai et al. 2005; Rivas-Carrillo et al. 2006; Uonaga et al. 2010; Zhang et al. 2004). Stimulation by VEGF alone has been shown to lead to the formation of leaky, thin-walled, immature vessels (Kenneth Ward 2008), while a combination of VEGF and other GFs (Golocheikine et al. 2010; Nillesen et al. 2006), including PDGF (Hao et al. 2007), has shown to be more beneficial. However, large amounts of GFs are needed to achieve benefits, which raises concerns on safety and cost-effectiveness of the approach. Growth factory controlled delivery through biomaterial interactions has shown benefits for islet transplantation (Stendahl et al. 
2008)(Vernon et al. 2012). In previous studies, we have shown that by delivery of a combination of VEGF and PDGF through a multifunctional recombinant fibronectin fragment that allows synergistic signaling between the GF receptors (through FNIII12-14) and the matrix-binding integrins domains (through the proximal FNIII9-10), the pro-angiogenic potential of the GFs is enhanced and GF doses can be substantially reduced. This was the rationale for utilizing the same strategy for engineering fibrin gels to provide prompt revascularization of islet grafts, which in turn promotes islet engraftment and function. We focused on this approach for GF delivery, rather than simple addition of GFs to fibrin matrices that would lead to burst release and would require higher amounts of GFs. While revascularization of islet grafts normally occurs two weeks after transplantation (Jansson and Carlsson 2002; Vasir et al. 2001), our engineered fibrin matrices allowed neogenesis of vascular beds early as 7 days after transplantation in both the SC and the EFP sites. Though we haven't evaluated whether islet-associated blood vessels had reconnected with the host vasculature, we hypothesize that the benefits in blood glucose control observed in the engineered firbin matrix conditions suggest a connection between islet newly formed microvessels and the host. Earlier and better revascularization may explain why we observed that engineered fibrin gels favored islet engraftment (reversal of diabetes) posttransplant and long-term function in controlling euglycemia of transplanted mice. Since we performed most studies with doses of islets that are considered marginal for the specific transplant sites, as expected, we did not observe reversal of diabetes within 7 days, despite enhanced revascularization. Additionally, we found that matrix stability in vivo, which we prolonged by addition of aprotinin, is critical for exploiting the beneficial effect of matrix-bound GFs, likely by prolonging GF bioavailability in the peri-transplant space around the islets, which outweights the decrease in cell infiltration.

This article is protected by copyright. All rights reserved 
In conclusion, our data support the hypothesis that biodegradable fibrin matrices engineered for controlled presentation and release of a combination of VEGF and PDGF and synergistic signaling between the corresponding GF receptors and integrins are beneficial for promoting islet engraftment and long-term function by inducing angiogenesis early after transplant in relevant extrahepatic transplant sites. Based on our previous findings in wound healing models (Martino et al. 2011), we suggest that the potent pro-angiogenic effects of engineered fibrin gels that lead to a more functional vessel morphology is mediated by the special synergistic signaling features of the FNIII9-10/12-14 recombinant protein that recruits and activate both endothelial cells and pericytes.

\section{Acknowledgments}

The authors are grateful to Celine Dessibourg for producing the FNIII9-10/12-14 recombinant protein, together with the Protein Expression Core Facility of EPFL, the members of the Diabetes Research Institute Preclinical Cell Processing and Translational Models Core for their help with surgical procedures, of the Imaging Core, and to Kevin Johnson for his most valuable help with histology. Funding was provided by philanthropic funds from the Diabetes Research Institute Foundation, grants from the Juvenile Diabetes Research Foundation (grant \# 17-2001268, 17-2010-5 and 17-2012-361), Converge Biotech, Inc. (Miami, FL, USA), the Fondazione IRCCS Ca' Granda Ospedale Maggiore Policlinico and the Swiss National Science Foundation.

This article is protected by copyright. All rights reserved 


\section{Disclosure}

The authors of this manuscript have no conflicts of interest to disclose as described by Biotechnology and Bioengineering.
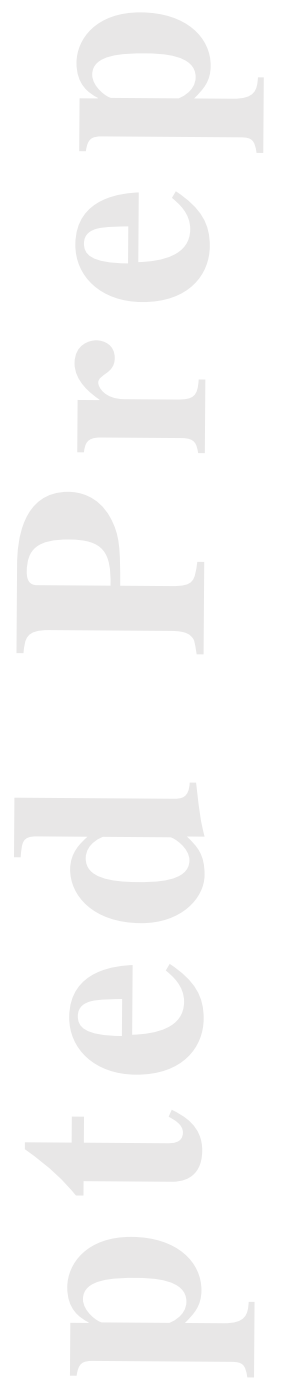

This article is protected by copyright. All rights reserved 


\section{References}

Ao Z, Matayoshi K, Lakey JR, Rajotte RV, Warnock GL. 1993. Survival and function of purified islets in the omental pouch site of outbred dogs. Transplantation 56(3):524-9.

Barkai U, Weir GC, Colton CK, Ludwig B, Bornstein SR, Brendel MD, Neufeld T, Bremer C, Leon A, Evron Y and others. 2012. Enhanced Oxygen Supply Improves Islet Viability in a New Bioartificial Pancreas. Cell Transplant.

Barshes NR, Wyllie S, Goss JA. 2005. Inflammation-mediated dysfunction and apoptosis in pancreatic islet transplantation: implications for intrahepatic grafts. J Leukoc Biol 77(5):587-97.

Beattie GM, Montgomery AM, Lopez AD, Hao E, Perez B, Just ML, Lakey JR, Hart ME, Hayek A. 2002. A novel approach to increase human islet cell mass while preserving beta-cell function. Diabetes 51(12):3435-9.

Berman DM, O'Neil JJ, Coffey LC, Chaffanjon PC, Kenyon NM, Ruiz P, Jr., Pileggi A, Ricordi C, Kenyon NS. 2009. Long-term survival of nonhuman primate islets implanted in an omental pouch on a biodegradable scaffold. Am J Transplant 9(1):91-104.

Brissova M, Shostak A, Shiota M, Wiebe PO, Poffenberger G, Kantz J, Chen Z, Carr C, Jerome WG, Chen J and others. 2006. Pancreatic islet production of vascular endothelial growth factor--a is essential for islet vascularization, revascularization, and function. Diabetes 55(11):2974-85.

Cantarelli E, Piemonti L. 2011. Alternative transplantation sites for pancreatic islet grafts. Curr Diab Rep 11(5):364-74.

Colton CK. 1995. Implantable biohybrid artificial organs. Cell Transplant 4(4):415-36.

Eming SA, Hubbell JA. 2011. Extracellular matrix in angiogenesis: dynamic structures with translational potential. Exp Dermatol 20(7):605-13.

Gibly RF, Graham JG, Luo X, Lowe WL, Jr., Hering BJ, Shea LD. 2011. Advancing islet transplantation: from engraftment to the immune response. Diabetologia 54(10):2494505.

Gill RG, Bishop NH. 2012. Clinical islet transplantation: where immunity and metabolism intersect? Curr Opin Endocrinol Diabetes Obes 19(4):249-54.

Golocheikine A, Tiriveedhi V, Angaswamy N, Benshoff N, Sabarinathan R, Mohanakumar T. 2010. Cooperative signaling for angiogenesis and neovascularization by VEGF and HGF following islet transplantation. Transplantation 90(7):725-31.

Gu Y, Tabata Y, Kawakami Y, Balamurugan AN, Hori H, Nagata N, Satake A, Cui W, Qi M, Misawa Y and others. 2001. Development of a new method to induce angiogenesis at subcutaneous site of streptozotocin-induced diabetic rats for islet transplantation. Cell Transplant 10(4-5):453-7.

Gustavson SM, Rajotte RV, Hunkeler D, Lakey JR, Edgerton DS, Neal DW, Snead WL, Penaloza AR, Cherrington AD. 2005. Islet auto-transplantation into an omental or splenic site results in a normal beta cell but abnormal alpha cell response to mild non-insulininduced hypoglycemia. Am J Transplant 5(10):2368-77.

Hao X, Silva EA, Mansson-Broberg A, Grinnemo KH, Siddiqui AJ, Dellgren G, Wardell E, Brodin LA, Mooney DJ, Sylven C. 2007. Angiogenic effects of sequential release of VEGF-A165 and PDGF-BB with alginate hydrogels after myocardial infarction. Cardiovasc Res 75(1):178-85.

This article is protected by copyright. All rights reserved 
Harlan DM, Kenyon NS, Korsgren O, Roep BO. 2009. Current advances and travails in islet transplantation. Diabetes 58(10):2175-84.

Hussey AJ, Winardi M, Han XL, Thomas GP, Penington AJ, Morrison WA, Knight KR, Feeney SJ. 2009. Seeding of pancreatic islets into prevascularized tissue engineering chambers. Tissue Eng Part A 15(12):3823-33.

Jacobs-Tulleneers-Thevissen D, Bartholomeus K, Suenens K, Vermeulen I, Ling Z, Hellemans KH, In't Veld P, Pipeleers-Marichal M, Pipeleers D. 2010. Human islet cell implants in a nude rat model of diabetes survive better in omentum than in liver with a positive influence of beta cell number and purity. Diabetologia 53(8):1690-9.

Jalili RB, Moeen Rezakhanlou A, Hosseini-Tabatabaei A, Ao Z, Warnock GL, Ghahary A. 2011. Fibroblast populated collagen matrix promotes islet survival and reduces the number of islets required for diabetes reversal. J Cell Physiol 226(7):1813-9.

Jansson L, Carlsson PO. 2002. Graft vascular function after transplantation of pancreatic islets. Diabetologia 45(6):749-63.

Johnson PR, Jones KE. 2012. Pancreatic islet transplantation. Semin Pediatr Surg 21(3):272-80.

Juang JH, Bonner-Weir S, Ogawa Y, Vacanti JP, Weir GC. 1996. Outcome of subcutaneous islet transplantation improved by polymer device. Transplantation 61(11):1557-61.

Juang JH, Hsu BR, Kuo CH. 2005. Islet transplantation at subcutaneous and intramuscular sites. Transplant Proc 37(8):3479-81.

Kenneth Ward W. 2008. A review of the foreign-body response to subcutaneously-implanted devices: the role of macrophages and cytokines in biofouling and fibrosis. J Diabetes Sci Technol 2(5):768-77.

Kim HI, Yu JE, Park CG, Kim SJ. 2010. Comparison of four pancreatic islet implantation sites. J Korean Med Sci 25(2):203-10.

Kim JS, Lim JH, Nam HY, Lim HJ, Shin JS, Shin JY, Ryu JH, Kim K, Kwon IC, Jin SM and others. 2012. In situ application of hydrogel-type fibrin-islet composite optimized for rapid glycemic control by subcutaneous xenogeneic porcine islet transplantation. $\mathbf{J}$ Control Release 162(2):382-90.

Kin T, Korbutt GS, Rajotte RV. 2003. Survival and metabolic function of syngeneic rat islet grafts transplanted in the omental pouch. Am J Transplant 3(3):281-5.

Kriz J, Vilk G, Mazzuca DM, Toleikis PM, Foster PJ, White DJ. 2012. A novel technique for the transplantation of pancreatic islets within a vascularized device into the greater omentum to achieve insulin independence. Am J Surg 203(6):793-7.

Lai Y, Schneider D, Kidszun A, Hauck-Schmalenberger I, Breier G, Brandhorst D, Brandhorst H, Iken M, Brendel MD, Bretzel RG and others. 2005. Vascular endothelial growth factor increases functional beta-cell mass by improvement of angiogenesis of isolated human and murine pancreatic islets. Transplantation 79(11):1530-6.

MacGregor RR, Williams SJ, Tong PY, Kover K, Moore WV, Stehno-Bittel L. 2006. Small rat islets are superior to large islets in in vitro function and in transplantation outcomes. Am J Physiol Endocrinol Metab 290(5):E771-9.

Martino MM, Hubbell JA. 2010. The 12th-14th type III repeats of fibronectin function as a highly promiscuous growth factor-binding domain. FASEB J 24(12):4711-21.

Martino MM, Mochizuki M, Rothenfluh DA, Rempel SA, Hubbell JA, Barker TH. 2009. Controlling integrin specificity and stem cell differentiation in 2D and 3D environments through regulation of fibronectin domain stability. Biomaterials 30(6):1089-97.

This article is protected by copyright. All rights reserved 
Martino MM, Tortelli F, Mochizuki M, Traub S, Ben-David D, Kuhn GA, Muller R, Livne E, Eming SA, Hubbell JA. 2011. Engineering the growth factor microenvironment with fibronectin domains to promote wound and bone tissue healing. Sci Transl Med 3(100):100ra89.

Marzorati S, Bocca N, Molano RD, Hogan AR, Doni M, Cobianchi L, Inverardi L, Ricordi C, Pileggi A. 2009. Effects of systemic immunosuppression on islet engraftment and function into a subcutaneous biocompatible device. Transplant Proc 41(1):352-3.

McQuilling JP, Arenas-Herrera J, Childers C, Pareta RA, Khanna O, Jiang B, Brey EM, Farney AC, Opara EC. 2011. New alginate microcapsule system for angiogenic protein delivery and immunoisolation of islets for transplantation in the rat omentum pouch. Transplant Proc 43(9):3262-4.

Mineo D, Pileggi A, Alejandro R, Ricordi C. 2009. Point: steady progress and current challenges in clinical islet transplantation. Diabetes Care 32(8):1563-9.

Nillesen ST, Geutjes PJ, Wismans R, Schalkwijk J, Daamen WF, van Kuppevelt TH. 2006. Increased angiogenesis in acellular scaffolds by combined release of FGF2 and VEGF. J Control Release 116(2):e88-90.

Nishimura R, Nishioka S, Fujisawa I, Shiku H, Shimada M, Sekiguchi S, Fujimori K, Ushiyama A, Matsue T, Ohuchi N and others. 2013. Tacrolimus inhibits the revascularization of isolated pancreatic islets. PLoS One 8(4):e56799.

Ohmura Y, Tanemura M, Kawaguchi N, Machida T, Tanida T, Deguchi T, Wada H, Kobayashi S, Marubashi S, Eguchi H and others. 2010. Combined transplantation of pancreatic islets and adipose tissue-derived stem cells enhances the survival and insulin function of islet grafts in diabetic mice. Transplantation 90(12):1366-73.

Pedraza E, Brady AC, Fraker CA, Molano RD, Sukert S, Berman DM, Kenyon NS, Pileggi A, Ricordi C, Stabler CL. 2012. Macroporous Three Dimensional PDMS Scaffolds for Extrahepatic Islet Transplantation. Cell Transplant.

Phelps EA, Headen DM, Taylor WR, Thule PM, Garcia AJ. 2013. Vasculogenic bio-synthetic hydrogel for enhancement of pancreatic islet engraftment and function in type 1 diabetes. Biomaterials 34(19):4602-11.

Pileggi A, Molano RD, Ricordi C, Zahr E, Collins J, Valdes R, Inverardi L. 2006. Reversal of diabetes by pancreatic islet transplantation into a subcutaneous, neovascularized device. Transplantation 81(9):1318-24.

Plesner A, Verchere CB. 2011. Advances and challenges in islet transplantation: islet procurement rates and lessons learned from suboptimal islet transplantation. J Transplant 2011:979527.

Rajab A. 2010. Islet transplantation: alternative sites. Curr Diab Rep 10(5):332-7.

Rickels MR. 2012. Recovery of endocrine function after islet and pancreas transplantation. Curr Diab Rep 12(5):587-96.

Rivas-Carrillo JD, Navarro-Alvarez N, Soto-Gutierrez A, Okitsu T, Chen Y, Tabata Y, Misawa $\mathrm{H}$, Noguchi H, Matsumoto S, Tanaka $\mathrm{N}$ and others. 2006. Amelioration of diabetes in mice after single-donor islet transplantation using the controlled release of gelatinized FGF-2. Cell Transplant 15(10):939-44.

Salvay DM, Rives CB, Zhang X, Chen F, Kaufman DB, Lowe WL, Jr., Shea LD. 2008. Extracellular matrix protein-coated scaffolds promote the reversal of diabetes after extrahepatic islet transplantation. Transplantation 85(10):1456-64.

This article is protected by copyright. All rights reserved 
Shapiro AM. 2011. Strategies toward single-donor islets of Langerhans transplantation. Curr Opin Organ Transplant 16(6):627-31.

Stendahl JC, Wang LJ, Chow LW, Kaufman DB, Stupp SI. 2008. Growth factor delivery from self-assembling nanofibers to facilitate islet transplantation. Transplantation 86(3):47881.

Stokes RA, Cheng K, Deters N, Lau SM, Hawthorne WJ, O'Connell PJ, Stolp J, Grey S, Loudovaris T, Kay TW and others. 2013. Hypoxia-inducible factor-1alpha (HIF-1alpha) potentiates beta-cell survival after islet transplantation of human and mouse islets. Cell Transplant 22(2):253-66.

Tavakoli A, Liong S. 2012. Pancreatic transplant in diabetes. Adv Exp Med Biol 771:420-37.

Uonaga T, Toyoda K, Okitsu T, Zhuang X, Yamane S, Uemoto S, Inagaki N. 2010. FGF-21 enhances islet engraftment in mouse syngeneic islet transplantation model. Islets 2(4):247-51.

van der Windt DJ, Echeverri GJ, Ijzermans JN, Cooper DK. 2008. The choice of anatomical site for islet transplantation. Cell Transplant 17(9):1005-14.

Vasir B, Jonas JC, Steil GM, Hollister-Lock J, Hasenkamp W, Sharma A, Bonner-Weir S, Weir GC. 2001. Gene expression of VEGF and its receptors Flk-1/KDR and Flt-1 in cultured and transplanted rat islets. Transplantation 71(7):924-35.

Vernon RB, Preisinger A, Gooden MD, D'Amico LA, Yue BB, Bollyky PL, Kuhr CS, Hefty TR, Nepom GT, Gebe JA. 2012. Reversal of diabetes in mice with a bioengineered islet implant incorporating a type I collagen hydrogel and sustained release of vascular endothelial growth factor. Cell Transplant 21(10):2099-110.

Wang W, Gu Y, Tabata Y, Miyamoto M, Hori H, Nagata N, Touma M, Balamurugan AN, Kawakami Y, Nozawa M and others. 2002. Reversal of diabetes in mice by xenotransplantation of a bioartificial pancreas in a prevascularized subcutaneous site. Transplantation 73(1):122-9.

Zhang N, Richter A, Suriawinata J, Harbaran S, Altomonte J, Cong L, Zhang H, Song K, Meseck M, Bromberg J and others. 2004. Elevated vascular endothelial growth factor production in islets improves islet graft vascularization. Diabetes 53(4):963-70.

This article is protected by copyright. All rights reserved 


\section{Tables}

Table I: Schematic of experimental groups for transplantation in the subcutaneous (SC) and the epididymal fat pad (EFP) sites

\section{Figure Legends}

Figure 1: Optimization of the subcutaneous site for islet transplantation. (A) Image of $80 \mu 1$ fibrin gels containing 1000 IEQ and polymerized on a sterile metal spatula used for subcutaneous (SC) transplantation. Scale bar: $7 \mathrm{~mm}$. (B) Percentage of chemically-induced diabetic C57BL/6 mice that became euglycemic as a function of time following transplantation of 1000 IEQ syngeneic islets in the SC site for each experimental group: islets transplanted alone (ISL AL, black, MRT: undefined, $n=5$ ), islets transplanted within fibrin gels with bound FNIII9-10/12-14 (ISL+FIB mix, green, MRT: undefined, $\mathrm{n}=5$ ), islets transplanted within fibrin gels with bound FNIII9-10/12-14 and VEGF-A165 + PDGF-BB with aprotinin (ISL+FIB+GF mix, red, MRT: 38 days, $\mathrm{n}=5$ ) and islets transplanted within fibrin gels with bound FNIII9-10/12-14 and VEGFA165 and PDGF-BB without aprotinin (ISL+FIB+GF w/o APR mix, pink, MRT: 84 days, n=3).

(C-F) Blood glucose (mg/dL) of diabetic mice transplanted with 1000 IEQ syngeneic islets in the SC site for each experimental group.

Figure 2: Characterization of islet subcutaneous syngeneic grafts at $7 \& 21$ days after transplant. Frozen sections $(75 \mu \mathrm{m}$ thick for day 7 and $5 \mu \mathrm{m}$ thick for day 21$)$ of subcutaneous (SC) tissue from C57BL/6 mice transplanted with 450 IEQ from syngeneic mice either alone 
(A), within fibrin gels with bound FNIII9-10/12-14 (B), or within fibrin gels with bound FNIII910/12-14 and VEGF-A165 + PDGF-BB with aprotinin (C,D) at day 7 (A-C) and at day 21 (D) after transplant. Representative images are shown of $n=3$. Sections were stained either for $H \& E$ (top row, light microscopy), or for insulin (INS: red), glucagon (GLU: cyan) and CD31 (green) (middle row, confocal microcopy), or for insulin (INS: red), Lyve-1 (green) and CD31 (cyan) (bottom row, confocal microcopy). $\mathrm{INS}^{+}$area and $\mathrm{CD} 1^{+}$area within $\mathrm{INS}^{+}$area within total graft area 7 days after transplant was quantified (pie charts: average values from surviving grafts from each group are shown). Samples in which islets were not found (most ISL AL and ISL+FIB mix) were not quantified. For comparison, control skin (E) and native normal pancreas (F) are shown. Scale bars: H\&E images $500 \mu \mathrm{m}$; fluorescence immunostaining images $50 \mu \mathrm{m} . \mathrm{n}=3$.

Figure 3: Characterization of endothelial-like cell outgrowth from ex vivo culture of islets within fibrin matrices. (A) Phase contrast images of islets cultured ex vivo either alone (ISL AL) or within fibrin gels with bound FNIII9-10/12-14 (ISL+FIB mix) or within fibrin gels with bound FNIII9-10/12-14 and VEGF-A165 + PDGF-BB with (ISL+FIB+GF mix) or without (ISL+FIB+GF w/o APR mix) aprotinin analyzed at day 2 (top row), day 3 ( $2^{\text {nd }}$ row), day 5 ( $3^{\text {rd }}$ row) and at day 11 (last row) after seeding. n=8-16. Scale bar: $500 \mu \mathrm{m}$. (B) Quantification of percentage of area occupied by cells outgrowing from cultured islets for each experimental condition. Day 2, 3, 5, 11: 'ISL AL' vs. 'ISL+FIB' p>0.05; 'ISL AL' and 'ISL+FIB' vs. 'ISL+FIB+GF' p<0.01; 'ISL AL' and 'ISL+FIB' vs. 'ISL+FIB+GF w/o APR' p<0.05; day 5: 'ISL+FIB+GF' vs. 'ISL+FIB+GF w/o APR' p<0.05.

This article is protected by copyright. All rights reserved 
Figure 4: Optimization of the epididymal fat pad site for islet transplantation in syngeneic islet transplants. (A) Percentage of euglycemic mice as a function of time after transplantation of 250 IEQ syngeneic islets into chemically-induced diabetic C57BL/6 mice in the EFP for each experimental group: islets transplanted alone (ISL AL, black) (MRT: 86 days, $n=8$ ), islets transplanted within fibrin gels with bound FNIII9-10/12-14 (ISL+FIB mix: green) (MRT: undefined, $n=5$ ), islets transplanted directly on the EFP and covered by fibrin gels with bound FNIII9-10/12-14 (ISL+FIB top: orange) (MRT: 54 days, n=5), islets transplanted within fibrin gels with bound FNIII9-10/12-14 and VEGF-A165 + PDGF-BB with aprotinin (ISL+FIB+GF mix: C,D, red) (MRT: undefined, $n=5$ ), islets transplanted directly on the EFP and covered by fibrin gels with bound FNIII9-10/12-14 and VEGF-A165 + PDGF-BB with aprotinin (ISL+FIB+GF top: blue) (MRT 38 days, $\mathrm{n}=10$ ). $\mathrm{p} \leq 0.05$ for ISL+FIB+GF top vs. vs ISL AL and ISL+FIB+GF mix. (B-F) Blood glucose (mg/dL) of diabetic mice transplanted with 250 IEQ syngeneic islets in the EFP pouch site for each experimental group. Graft-containing EFPs were removed at day 112 to confirm graft function (arrows). Representative images are shown of $\mathrm{n}=10$ (G) Formalin-fixed paraffin-embedded $5 \mu \mathrm{m}$ thick sections of EFP tissue from C57BL/6 mice transplanted with 250 IEQ syngeneic islets directly on the EFP and covered with fibrin gels with bound FNIII9-10/12-14 and VEGF-A165 + PDGF-BB with aprotinin (ISL+FIB+GF top) at day 112 after transplant were stained either for H\&E (left column, light microscopy), or for insulin (INS: red) and glucagon (GLU: green) (2 $2^{\text {nd }}$ column, confocal microcopy), or for CD31 (green) and insulin (INS: red) $\left(3^{\text {rd }}\right.$ column, confocal microcopy), or for Lyve-1 (green) and insulin (INS: red) (last column, confocal microcopy) and compared to pancreas and control EFP. (H) Quantification of percentages of $\mathrm{CD} 1^{+}$area within the grafts of the ISL+FIB+GF top condition at day 112 after transplant and compared to control pancreas. $\mathrm{n}=10$ mice.

This article is protected by copyright. All rights reserved 
Figure 5: Characterization of the epididymal fat pad site for islet transplantation in syngeneic and allogeneic islet transplants. (A-D) Blood glucose $(\mathrm{mg} / \mathrm{dL}, \mathrm{A})$ of diabetic mice transplanted with 1000 IEQ syngeneic islets in the optimized EFP site (ISL+FIB+GF top) and analyzed histologically and through immunofluorescence for islet phenotype (insulin: red and glucagon: green) and re-vascularization (CD31, green) (formalin-fixed paraffin-embedded $5 \mu \mathrm{m}$ thick sections of EFP tissue) at day 7 (B), 14 (C) and 21 (D) after transplant. Representative images are shown of $n=3$. $(E, F)$ Blood glucose $(\mathrm{mg} / \mathrm{dL}, \mathrm{E})$ of diabetic BALB/c mice that became euglycemic after transplant of different doses (750 IEQ: orange, $n=2 ; 1000$ IEQ: red, $n=6 ; 1200$ I EQ: black, $n=7$ ) of islets from fully MHC-mismatched C57BL/6 mice in the optimized EFP site (ISL+FIB+GF top); $\mathrm{p}<0.001$.

This article is protected by copyright. All rights reserved 


\section{Table 1.}

SC site

\begin{tabular}{|c|c|c|c|c|}
\hline $\begin{array}{c}\text { Experimental } \\
\text { groups }\end{array}$ & ISL AL & $\begin{array}{c}\text { ISL + FIB } \\
\text { mix }\end{array}$ & $\begin{array}{c}\text { ISL }+ \text { FIB }+ \text { GF } \\
\text { mix }\end{array}$ & $\begin{array}{c}\text { ISL + FIB + GF } \\
\text { w/o APR mix }\end{array}$ \\
\hline Fibrin gel & & $\mathrm{x}$ & $x$ & $x$ \\
\hline FN 9-10/12-14 & & $\mathrm{x}$ & $\mathrm{x}$ & $\mathrm{x}$ \\
\hline VEGF-A165 & & & $\mathrm{x}$ & $\mathrm{x}$ \\
\hline PDGF-BB & & & $\mathrm{x}$ & $\mathrm{x}$ \\
\hline Aprotinin & & $\mathrm{x}$ & $\mathrm{x}$ & \\
\hline
\end{tabular}

\section{EFP site}

\begin{tabular}{|l|c|c|c|c|c|}
\hline $\begin{array}{c}\text { Experimental } \\
\text { groups }\end{array}$ & ISL AL & ISL + FIB & ISL + FIB top & $\begin{array}{c}\text { ISL + FIB + GF } \\
\text { mix }\end{array}$ & ISL + FIB + GF \\
\hline Fibrin gel & & $\mathrm{x}$ & $\mathrm{x}$ & $\mathrm{x}$ & $\mathrm{x}$ \\
\hline FN 9-10 12-14 & & $\mathrm{x}$ & $\mathrm{x}$ & $\mathrm{x}$ & $\mathrm{x}$ \\
\hline VEGF-A165 & & & & $\mathrm{x}$ & $\mathrm{x}$ \\
\hline PDGF-BB & & & & $\mathrm{x}$ & $\mathrm{x}$ \\
\hline Aprotinin & & $\mathrm{x}$ & $\mathrm{x}$ & $\mathrm{x}$ & $\mathrm{x}$ \\
\hline
\end{tabular}

This article is protected by copyright. All rights reserved 

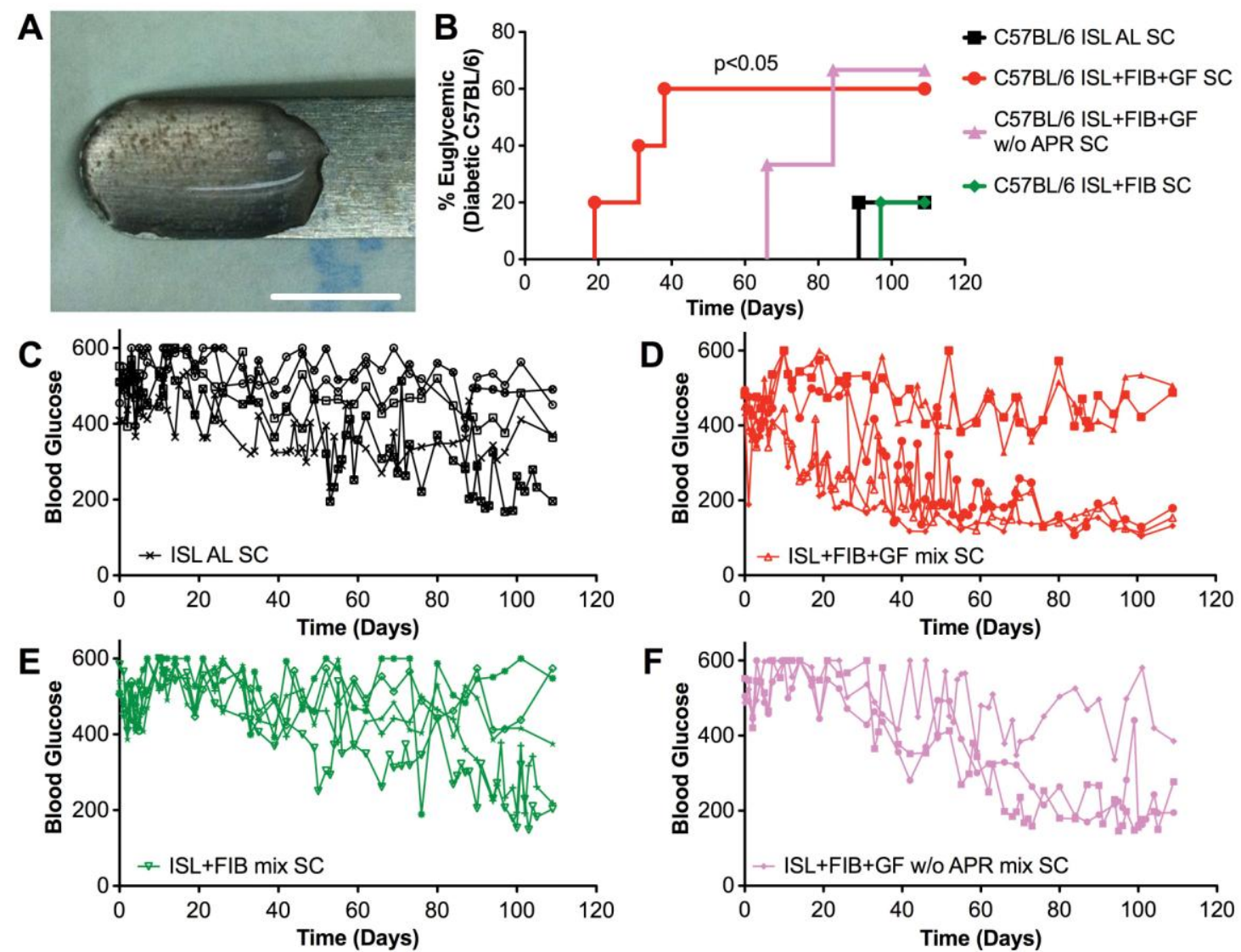

Figure 1

This article is protected by copyright. All rights reserved 


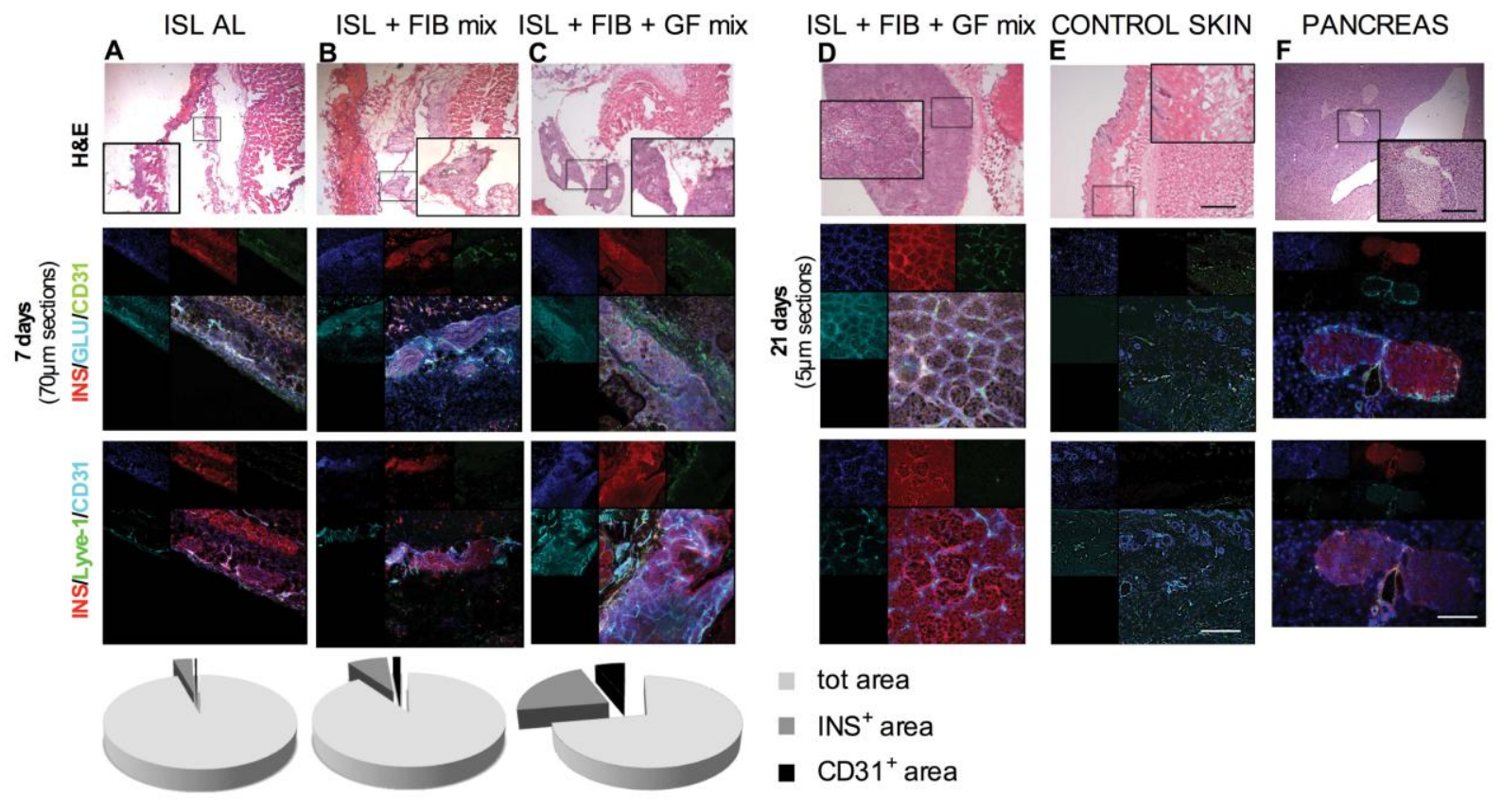

Figure 2

This article is protected by copyright. All rights reserved 


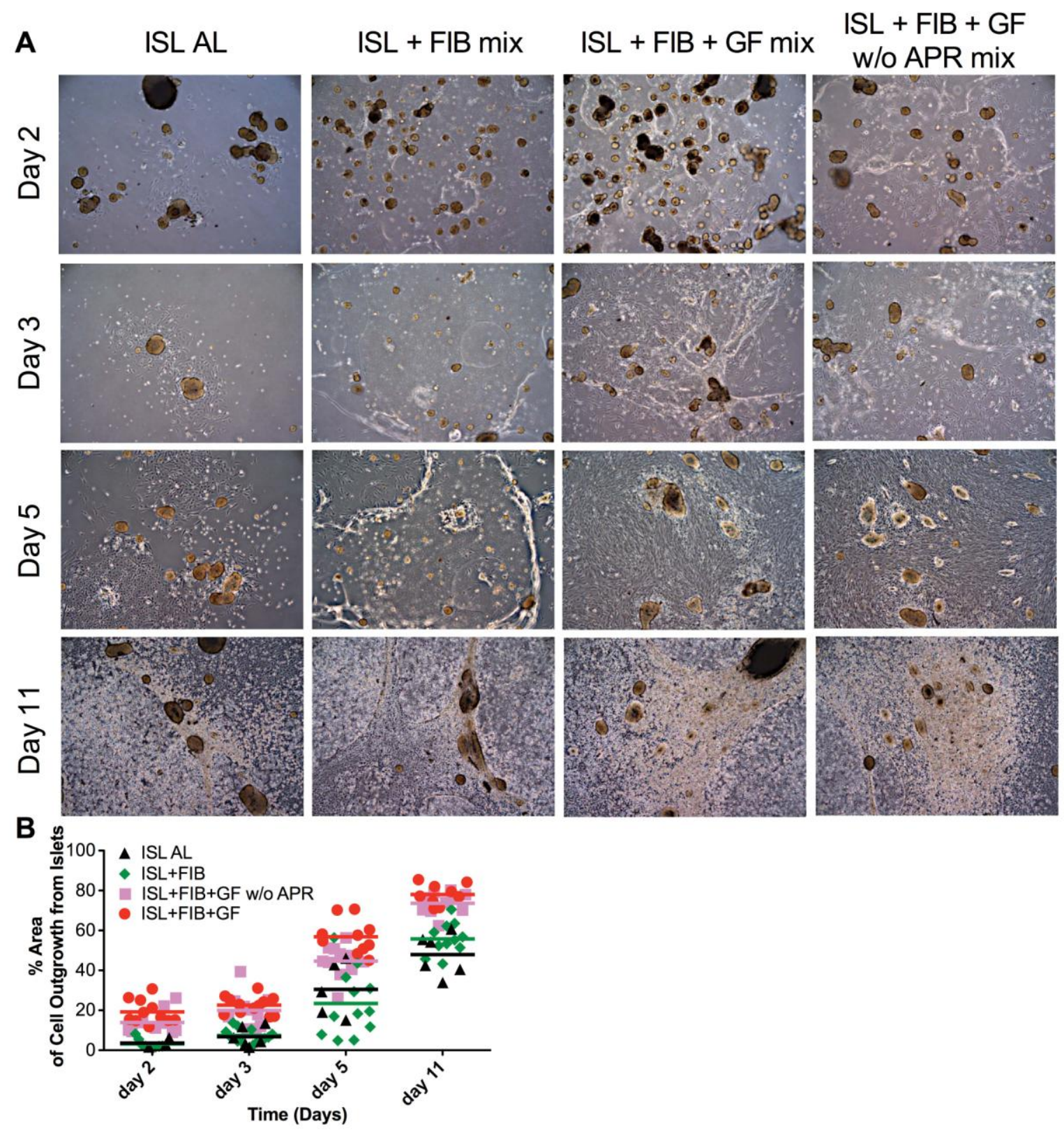

Figure 3

This article is protected by copyright. All rights reserved 

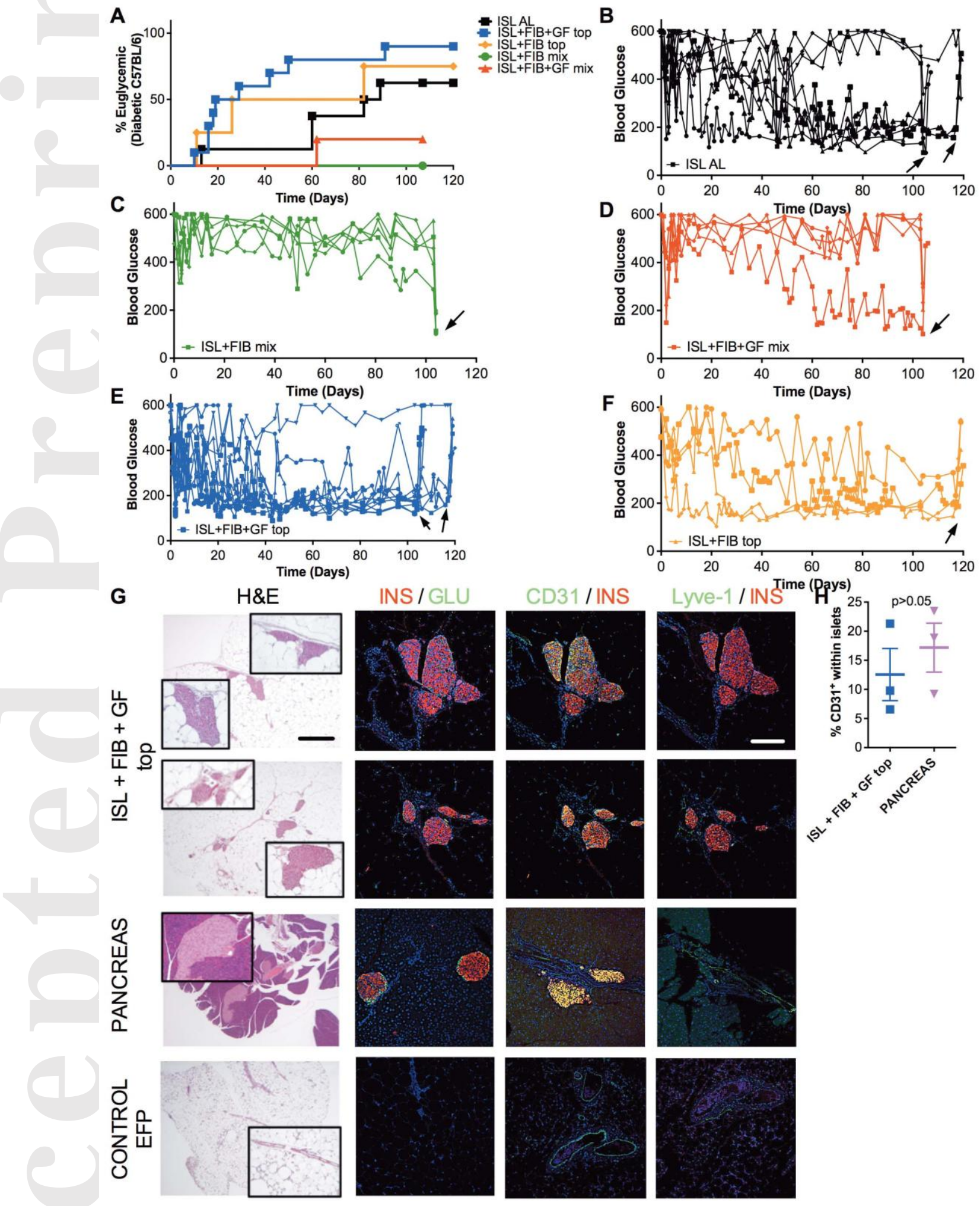

Figure 4

This article is protected by copyright. All rights reserved 
A
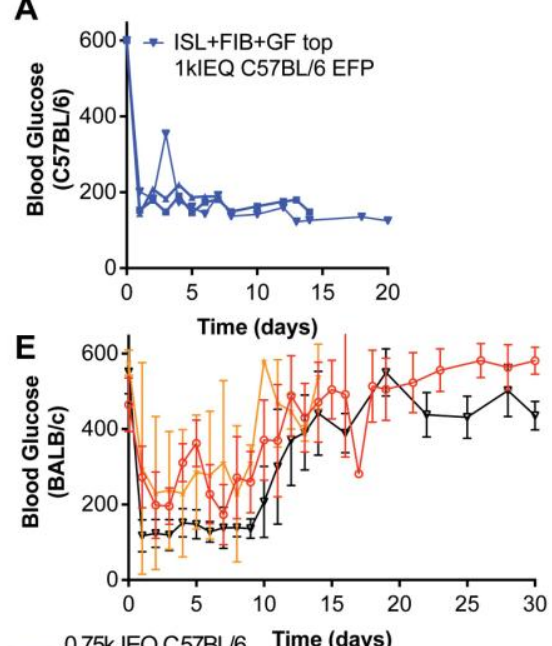

$-\underset{\text { EFP }}{0.75 k}$

- $1 \mathrm{k}$ IEQ C57BL6

$-\underset{\text { EFP }}{1.2 \mathrm{k} \text { IEQ C57BL6 }}$
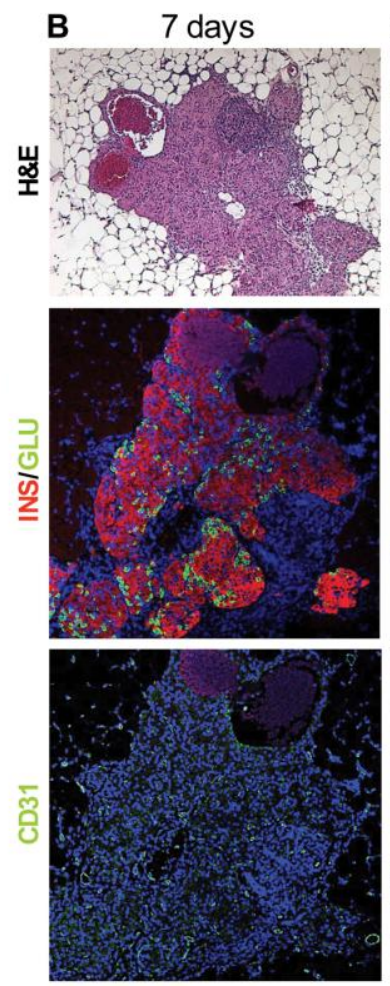

C 14 days

D 21 days
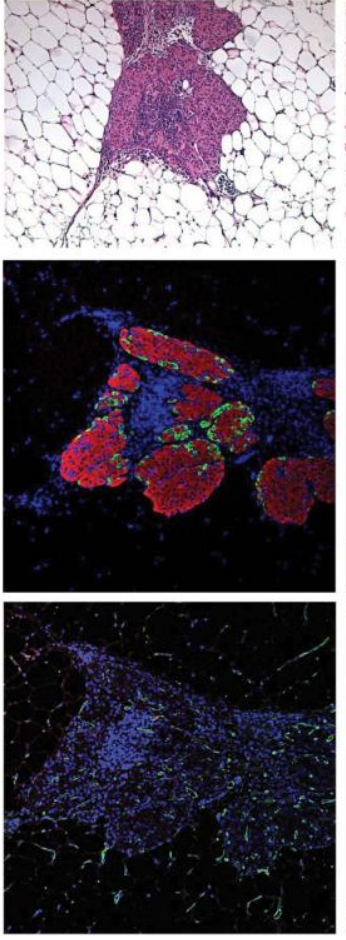

Figure 5
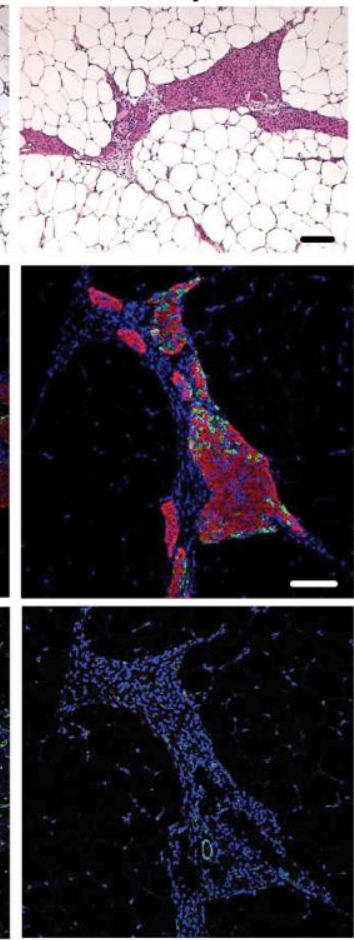

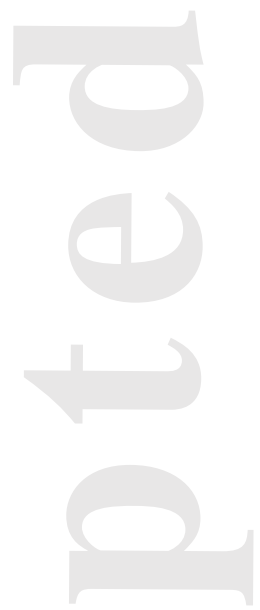

This article is protected by copyright. All rights reserved 